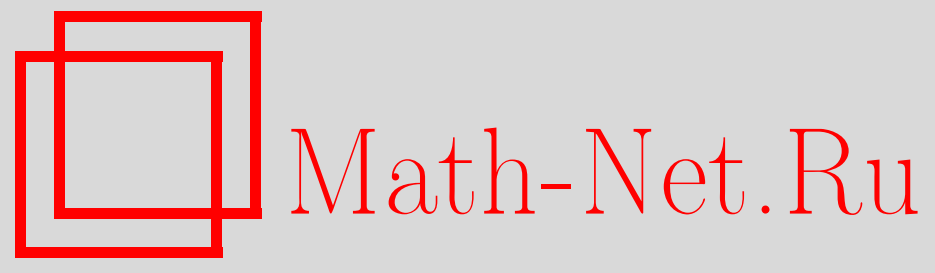

В. А. Лукьянов, Л. Н. Кривоносов, Уравнения Янга-Миллса на 4-многообразиях конформной связности без кручения с различными сигнатурами, Вестн. Сам. гос. техн. ун-та. Сер. Физ.-мат. науки, 2017, номер 4, 633-650

DOI: https://doi.org/10.14498/vsgtu1562

Использование Общероссийского математического портала MathNet.Ru подразумевает, что вы прочитали и согласны с пользовательским соглашением

http://www.mathnet.ru/rus/agreement

Параметры загрузки:

IP : 54.197 .130 .99

26 апреля 2023 г., 13:39:04




Вестн. Сам. гос. техн. ун-та. Сер. Физ.-мат. науки. 2017. Т. 21, № 4 . С. $633-650$ ISSN: 2310-7081 (online), 1991-8615 (print)

УДК 514.756

\title{
Уравнения Янга-Миллса на 4-многообразиях конформной связности без кручения с различными сигнатурами
}

\author{
Л. Н. Кривоносов, В.А. Лукъянов
}

Нижегородский государственный технический университет им. Р. Е. Алексеева, Россия, 603600, Нижний Новгород, ул. Минина, 24.

\section{Аннотация}

Исследуются пространства конформной связности без кручения размерности 4, матрица связности которых удовлетворяет уравнениям ЯнгаМиллса. Здесь мы обобщаем и усиливаем результаты, полученные нами в предыдущих статьях, где угловая метрика этих пространств имела сигнатуру Минковского. Обобщение состоит в том, что здесь мы исследуем пространства всех возможных сигнатур метрики, а усиление связано с тем, что дополнительное внимание уделяется вычислению матрицы кривизны и установлению свойств ее компонент. Показано, что уравнения Янга-Миллса на 4-многообразиях конформной связности без кручения при произвольной сигнатуре угловой метрики сводятся к уравнениям Эйнштейна, уравнениям Максвелла и равенству тензора Баха угловой метрики и тензора энергии-импульса кососимметричного тензора заряда. Доказано, что в случае равенства нулю тензора Вейля уравнения Янга-Миллса имеют только автодуальные или антиавтодуальные решения, т.е. матрица кривизны конформной связности состоит из автодуальных или антиавтодуальных внешних 2-форм. При сигнатуре Минковского (анти)автодуальные внешние 2-формы могут быть лишь нулевыми. Вычислены компоненты матрицы кривизны в случае, когда угловая метрика произвольной сигнатуры является эйнштейновой, а связность удовлетворяет уравнениям Янга-Миллса. В евклидовом и псевдоевклидовом 4-пространствах приведены некоторые частные автодуальные и антиавтодуальные решения уравнений Максвелла, к которым сводятся в данном случае все уравнения Янга-Миллса.

Ключевые слова: многообразия конформной связности, кривизна, кручение, уравнения Янга-Миллса, уравнения Эйнштейна, уравнения Максвелла, оператор Ходжа, (анти)автодуальные 2-формы, тензор Вейля, тензор Баха.

\section{Научная статья}

2 (ㅇ) Контент публикуется на условиях лицензии Creative Commons Attribution 4.0 International (https://creativecommons.org/licenses/by/4.0/deed.ru)

\section{Образец для цитирования}

К р и в он о с о в Л. Н., Лу к ь я н в В. А. Уравнения Янга-Миллса на 4-многообразиях конформной связности без кручения с различными сигнатурами // Bестн. Сам. гос. техн. ун-та. Сер. Физ.-мат. науки, 2017. Т. 21, № 4. С. 633-650. doi: 10.14498/vsgtu1562.

\section{Сведения об авторах}

Вячеслав Анатольевич Лукъянов (10) http://orcid.org/0000-0002-7294-0232 кандидат физико-математических наук; доцент; каф. прикладной математики; e-mail: oxyzt@ya.ru

Леонид Николаевич Кривоносов (1) http://orcid.org/0000-0002-3533-9595 кандидат физико-математических наук, доцент; доцент; каф. прикладной математики; e-mail: 1.n.krivonosov@gmail.com 
Получение: 12 октября 2017 г. / Исправление: 27 ноября 2017 г. / Принятие: 18 декабря 2017 г. / Публикация онлайн: 28 декабря 2017 г.

Введение. Исследуются уравнения Янга-Миллса на 4-многообразиях конформной связности с нулевым кручением. Такие многообразия задаются матрицей $6 \times 6$ конформной связности

$$
\Omega=\left(\begin{array}{ccc}
\omega_{0}^{0} & \omega_{i} & 0 \\
\omega^{j} & \omega_{i}^{j} & -\eta^{j k} \omega_{k} \\
0 & -\eta_{i k} \omega^{k} & -\omega_{0}^{0}
\end{array}\right), \quad \eta_{i k} \omega_{j}^{k}+\eta_{j k} \omega_{i}^{k}=0
$$

на каждой карте некоторого атласа. Элементы этой матрицы являются пфаффовыми формами от координат карты. Индексы $i, j, k, l, p, q, m, n$ будут в дальнейшем принимать значения $1,2,3,4 ; \eta_{i j}=0$ при $i \neq j$, а $\eta_{i i}= \pm 1$. Квадратичная форма $\psi=\eta_{i j} \omega^{i} \omega^{j}$ называется угловой метрикой. Сигнату$p a s$ угловой метрики есть разность между числом положительных и отрицательных $\eta_{i i}$. На пересекающихся картах $U_{\alpha} \cap U_{\beta} \neq \varnothing$ должна быть задана матрица перехода $h_{\alpha \beta}$, которая связывает матрицы конформной связности $\Omega_{\alpha}$ и $\Omega_{\beta}$ следующей формулой:

$$
\Omega_{\beta}=\left(h_{\alpha \beta}\right)^{-1} d h_{\alpha \beta}+\left(h_{\alpha \beta}\right)^{-1} \Omega_{\alpha} h_{\alpha \beta} .
$$

Переходные матрицы $h$ принадлежат 11-параметрической группе $H_{4, s}$, которая является подгруппой стационарности конформной группы $C_{4, s}$. Группа $H_{4, s}$ называется калибровочной группой. На каждой карте $U_{\alpha}$ матрица связности задается с точностью до калибровочного преобразования $h$ и изменяется по закону, аналогичному (2):

$$
\widetilde{\Omega}_{\alpha}=h^{-1} d h+h^{-1} \Omega_{\alpha} h .
$$

С помощью калибровочного преобразования нормализации на каждой карте можно добиться $\omega_{0}^{0}=0$, что мы и будем всегда далее предполагать. Матрица конформной связности (1) порождает матрицу конформной кривизHol

$$
\Phi \stackrel{\text { def }}{=} d \Omega+\Omega \wedge \Omega
$$

Алгебраическая структура матрицы $\Phi$ такая же, как у $\Omega$ :

$$
\Phi=\left(\begin{array}{ccc}
\Phi_{0}^{0} & \Phi_{i} & 0 \\
\Phi^{j} & \Phi_{i}^{j} & -\eta^{j k} \Phi_{k} \\
0 & -\eta_{i k} \Phi^{k} & -\Phi_{0}^{0}
\end{array}\right), \quad \eta_{i k} \Phi_{j}^{k}+\eta_{j k} \Phi_{i}^{k}=0 .
$$

Из (4) и (1) с учетом $\omega_{0}^{0}=0$ имеем

$$
\begin{aligned}
\Phi_{0}^{0} & =\omega_{k} \wedge \omega^{k}, \\
\Phi_{i} & =d \omega_{i}+\omega_{k} \wedge \omega_{i}^{k}=\nabla \omega_{i}, \\
\Phi^{j} & =d \omega^{j}+\omega_{k}^{j} \wedge \omega^{k}=\nabla \omega^{j}, \\
\Phi_{i}^{j} & =d \omega_{i}^{j}+\omega_{k}^{j} \wedge \omega_{i}^{k}+\omega^{j} \wedge \omega_{i}+\eta^{j m} \eta_{i k} \omega_{m} \wedge \omega^{k}= \\
& =R_{i}^{j}+\omega^{j} \wedge \omega_{i}+\eta^{j m} \eta_{i k} \omega_{m} \wedge \omega^{k},
\end{aligned}
$$


где $R_{i}^{j}=\frac{1}{2} R_{i k l}^{j} \omega^{k} \wedge \omega^{l}$ - тензор кривизны квадратичной формы $\eta_{i j} \omega^{i} \omega^{j}$, а $R_{i k l}^{j}$ - тензор Римана.

Внешнее дифференцирование (4) приводит к тождествам Бианки

$$
d \Phi+\Omega \wedge \Phi-\Phi \wedge \Omega \equiv 0 .
$$

Внешнее дифференцирование (3) дает закон преобразования матрицы конформной кривизны на карте $U_{\alpha}$ относительно калибровочных преобразований $h$ :

$$
\widetilde{\Phi}_{\alpha}=h^{-1} \Phi_{\alpha} h
$$

Из $\eta_{i k}$ можно сконструировать величину

$$
\varepsilon_{i j}^{k l} \stackrel{\text { def }}{=} \delta_{1234}^{k l p q} \eta_{p i} \eta_{q j}
$$

где $\delta_{1234}^{k l p q}$ - символ Кронекера. Onератор Ходжа обозначается * и действует на внешнюю 2-форму $\theta=a_{i j} \omega^{i} \wedge \omega^{j}$ по правилу

$$
* \theta=\frac{1}{2} a_{i j} \varepsilon_{k l}^{i j} \omega^{k} \wedge \omega^{l} .
$$

Матрицы компонент $\eta_{i j}=\eta^{i j}$ для сигнатур $\pm 4, \pm 2,0$ имеют, соответственно, вид

$$
\left(\eta_{i j}\right)= \pm\left(\begin{array}{llll}
1 & 0 & 0 & 0 \\
0 & 1 & 0 & 0 \\
0 & 0 & 1 & 0 \\
0 & 0 & 0 & 1
\end{array}\right), \quad \pm\left(\begin{array}{cccc}
-1 & 0 & 0 & 0 \\
0 & 1 & 0 & 0 \\
0 & 0 & 1 & 0 \\
0 & 0 & 0 & 1
\end{array}\right), \quad\left(\begin{array}{cccc}
-1 & 0 & 0 & 0 \\
0 & -1 & 0 & 0 \\
0 & 0 & 1 & 0 \\
0 & 0 & 0 & 1
\end{array}\right)
$$

Ненулевые компоненты $\varepsilon_{i j}^{k l}(9)$ имеют вид:

$$
\begin{array}{clll}
\text { для } s= \pm 2- & \varepsilon_{12}^{34}=-1, & \varepsilon_{13}^{24}=1, & \varepsilon_{14}^{23}=-1, \\
& \varepsilon_{23}^{14}=1, & \varepsilon_{24}^{13}=-1, & \varepsilon_{34}^{12}=1 ; \\
\text { для } s= \pm 4- & \varepsilon_{12}^{34}=1, & \varepsilon_{13}^{24}=-1, & \varepsilon_{14}^{23}=1, \\
& \varepsilon_{23}^{14}=1, & \varepsilon_{24}^{13}=-1, & \varepsilon_{34}^{12}=1 ; \\
\text { для } s=0- & \varepsilon_{12}^{34}=1, & \varepsilon_{13}^{24}=1, & \varepsilon_{14}^{23}=-1, \\
& \varepsilon_{23}^{14}=-1, & \varepsilon_{24}^{13}=1, & \varepsilon_{34}^{12}=1 .
\end{array}
$$

2-форма $\theta$ называется автодуалъной (антиавтодуальной), если $* \theta=\theta$ $(* \theta=-\theta)$. Для $s= \pm 2$ имеем $*^{2}=-i d$, а для $s= \pm 4 ; 0$ будет $*^{2}=i d$. Это различие приводит к тому, что в случаях $s= \pm 4 ; 0$ существуют ненулевые автодуальные и антиавтодуальные 2-формы, а в случае $s= \pm 2$ их нет. Уравнением Янга-Миллса на 4-многообразии называется уравнение, аналогичное тождеству Бианки (7):

$$
d * \Phi+\Omega \wedge * \Phi-* \Phi \wedge \Omega=0
$$

2-формы $\Phi^{j}$, компоненты матрицы (5), образуют геометрический объект, так как при преобразованиях (8) 2-формы $\widetilde{\Phi}^{j}$ выражаются только через $\Phi^{j}$. 
Он называется кручением [1, стр. 169]. Мы изучаем уравнения Янга-Миллса только при нулевом кручении

$$
\Phi^{j}=0,
$$

так как при ненулевом кручении они слишком сложные.

При нулевом кручении 2-форма $\Phi_{0}^{0}$ также становится геометрическим объектом, который мы называем зарядом. В работе [2] уравнения Янга-Миллса изучались только при сигнатуре $s=2$. При $s=-2$ все результаты идентичны. Но при сигнатурах $s= \pm 4 ; 0$ ввиду наличия автодуальных (антиавтодуальных) 2-форм некоторые результаты оказываются существенно другими. В частности, в [2] было доказано, что когда тензор Вейля угловой метрики $\eta_{i j} \omega^{i} \omega^{j}$ равен нулю или когда угловая метрика эйнштейнова, электромагнитное поле (то есть заряд $\Phi_{0}^{0}=\frac{1}{2} b_{[i j]} \omega^{j} \wedge \omega^{i}$ ) при $s=2$ равно нулю. В настоящей статье мы эти результаты усиливаем, вычислив всю матрицу кривизны. При нулевом тензоре Вейля уравнения Янга-Миллса (13) выполняются только в случае нулевой матрицы кривизны, $\Phi=0$. А в случае эйнштейновой метрики матрица кривизны вычисляется только через тензор Вейля, подтверждая тем самым, что электромагнитное поле равно нулю.

При $s= \pm 4 ; 0$ уравнения Янга-Миллса в случае нулевого тензора Вейля или эйнштейновой угловой метрики имеют решения с ненулевым тензором заряда $b_{[i j]}$. В случае нулевого тензора Вейля вся матрица кривизны вычисляется через заряд, причем она автодуальна или антиавтодуальна, а уравнения Янга-Миллса сводятся к уравнениям Максвелла на компоненты тензора заряда $b_{[i j]}$. В случае эйнштейновой метрики матрица $\Phi$ вычисляется через тензор заряда и тензор Вейля. При этом 2-формы $\Phi_{0}^{0}$ и $\Phi_{i}$ автодуальны или антиавтодуальны. Но вся матрица кривизны этим условиям не удовлетворяет.

Отметим, что (анти)автодуальность на римановых 4-многообразиях изучалась многими авторами с разных точек зрения [3-5].

1. Уравнения Янга-Миллса. Подробная запись уравнений Янга-Миллса (13) в пространстве без кручения, при $\Phi^{j}=0$, такова:

$$
\begin{gathered}
d * \Phi_{0}^{0}-* \Phi_{k} \wedge \omega^{k}=0, \quad * \Phi_{0}^{0} \wedge \omega^{i}-* \Phi_{k}^{i} \wedge \omega^{k}=0, \\
\nabla * \Phi_{i}+\omega_{k} \wedge * \Phi_{i}^{k}-* \Phi_{0}^{0} \wedge \omega_{i}=0, \\
\nabla * \Phi_{i}^{j}+\omega^{j} \wedge * \Phi_{i}-\eta_{i n} \eta^{j m} * \Phi_{m} \wedge \omega^{n}=0 .
\end{gathered}
$$

В [2] была доказана главная формула пространства Янга-Миллса (многообразия конформной связности без кручения, в котором выполнены уравнения Янга-Миллса) при $s=2$ :

$$
* \Phi_{i}^{j}=\frac{1}{2} \varepsilon_{i q}^{j p} \Phi_{p}^{q}
$$

Эта формула верна и для всех остальных сигнатур, доказательство аналогичное. Как и в статье [2], с помощью (16) можно показать, что система (15) при любой сигнатуре эквивалентна

$$
\begin{gathered}
d \Phi_{0}^{0}=0, \quad d * \Phi_{0}^{0}=0 \\
* \Phi_{0}^{0} \wedge \omega^{j}-* \Phi_{k}^{j} \wedge \omega^{k}=0, \\
\nabla * \Phi_{i}+\omega_{k} \wedge * \Phi_{i}^{k}-* \Phi_{0}^{0} \wedge \omega_{i}=0 .
\end{gathered}
$$


Компонентная запись уравнений (18) имеет вид [2, стр. 438]

$$
\Phi_{i j}+b_{[i j]}=0,
$$

где

$$
\Phi_{i j} \stackrel{\text { def }}{=} \Phi_{i j k}^{k}, \quad \Phi_{i}^{j} \stackrel{\text { def }}{=} \frac{1}{2} \Phi_{i k l}^{j} \omega^{k} \wedge \omega^{l}, \quad \omega_{i} \stackrel{\text { def }}{=} b_{i k} \omega^{k}, \quad b_{[i j]} \stackrel{\text { def }}{=} b_{i j}-b_{j i} .
$$

Из $(6)_{4}$ и (21) следует

$$
\Phi_{i j}=R_{i j}-2 b_{i j}-b \eta_{i j},
$$

где $R_{i j} \stackrel{d e f}{=} R_{i j k}^{k}$ - тензор Риччи квадратичной формы угловой метрики $\psi=$ $=\eta_{i j} \omega^{i} \omega^{j}, b \stackrel{\text { def }}{=} \eta^{i j} b_{i j}$, поэтому равенство (20) можно переписать в виде

$$
R_{i j}=b_{(i j)}+b \eta_{i j}, \quad b_{(i j)} \stackrel{\text { def }}{=} b_{i j}+b_{j i},
$$

что представляет собой уравнения Эйнштейна. Свертка $(22)_{1}$ с $\eta^{i j}$ дает $R=$ $=6 b$. Таким образом, (18)- уравнения Эйнштейна, а (17) - уравнения Максвелла. Пространства конформной связности без кручения, где $\Phi_{0}^{0}=0$ и выполняется условие (20), Картан назвал нормальными [1, стр. 178].

Для уравнений (19) в [2, стр. 444] была получена компонентная запись

$$
\eta^{m n}\left(-b_{(i j) \mid m n}+b_{(p m)} R_{(i j) n}^{p}+2 b_{[i m]} b_{[j n]}\right)-2 b b_{(i j)}+b_{\mid(i j)}-2 Q \eta_{i j}=0,
$$

где $Q \stackrel{\text { def }}{=} \eta^{i j} \eta^{m n} b_{i m} b_{j n}$. Эта формула также верна для любых сигнатур. Так как в силу $(22)$ величины $b_{(i j)}$ выражаются только через тензор Риччи квадратичной формы угловой метрики $\psi$, для физических применений эту формулу целесообразно переписать, оставив слева только слагаемые, выражающиеся через $\psi$ :

$$
\begin{aligned}
\eta^{m n}\left(b_{(m p)} R_{(i j) n}^{p}-b_{(i j) \mid m n}\right)+b_{\mid(i j)} & -2 b b_{(i j)}-\frac{1}{2} \eta^{p q} \eta^{m n} b_{(p m)} b_{(q n)} \eta_{i j}= \\
= & \frac{1}{2} \eta_{i j} \eta^{p q} \eta^{m n} b_{[p m]} b_{[q n]}-2 \eta^{m n} b_{[i m]} b_{[j n]} .
\end{aligned}
$$

Здесь слева стоит тензор Баха $B_{i j}$ квадратичной формы угловой метрики $\psi$, а справа - тензор энергии-импульса внешней 2-формы $\Phi_{0}^{0}=\frac{1}{2} b_{[i j]} \omega^{j} \wedge \omega^{i}$, который мы определили по аналогии с тензором энергии-импульса для электромагнитного поля [6, формула 33,1]. Другая форма записи для тензора Баха:

$$
B_{i j}=2\left(\nabla^{k} \nabla_{k} P_{i j}-\nabla^{k} \nabla_{i} P_{k j}\right)+2 P^{k l} C_{i k j l},
$$

где $C_{i k j l}$ - тензор Вейля,

$$
P_{i j} \stackrel{\text { def }}{=} \frac{1}{12} R \eta_{i j}-\frac{1}{2} R_{i j}=-\frac{1}{2} b_{(i j)}
$$

(использовали $(22)$ и $R=6 b)$. Если тензор энергии-импульса обозначить через $T_{i j}$, то уравнение (23) (то же, что и (19)) запишется в виде

$$
B_{i j}=T_{i j} .
$$


2. Уравнения Янга-Миллса при нулевом тензоре Вейля. Всякий кососимметрический тензор $F_{i j}$ в псевдоевклидовом пространстве с метрическим тензором $\eta_{i j}$ может быть записан в виде

$$
\left(F_{i j}\right)=\left(\begin{array}{cccc}
0 & E_{1} & E_{2} & E_{3} \\
-E_{1} & 0 & -H_{3} & H_{2} \\
-E_{2} & H_{3} & 0 & -H_{1} \\
-E_{3} & -H_{2} & H_{1} & 0
\end{array}\right)
$$

По аналогии с электромагнитным полем тензором энергии-импульса кососимметрического тензора $F_{i j}$ назовем

$$
T_{i j} \stackrel{\text { def }}{=} \frac{1}{2} \eta_{i j} F^{p q} F_{p q}-2 \eta^{p q} F_{i p} F_{j q}
$$

При сигнатуре $s= \pm 2$ элемент $T_{11}$ имеет, соответственно, вид

$$
T_{11}=\mp\left(\left(E_{1}\right)^{2}+\left(E_{2}\right)^{2}+\left(E_{3}\right)^{2}+\left(H_{1}\right)^{2}+\left(H_{2}\right)^{2}+\left(H_{3}\right)^{2}\right) .
$$

Отсюда ясно, что из $T_{i j}=0$ (при всех $\left.i, j\right)$ следует $F_{i j}=0$. Но для других сигнатур это не так. Рассмотрим 2-форму

$$
F=\frac{1}{2} F_{i j} d x^{i} \wedge d x^{j}
$$

где $x^{1}, x^{2}, x^{3}, x^{4}$ - координаты псевдоевклидова пространства, а $F_{i j}$ задаются формулой (27).

Лемма. Для сигнатур $s= \pm 4 ; 0$ тензор $T_{i j}$ обращается в нуль тогда и только тогда, когда 2-форма $F=\frac{1}{2} F_{i j} d x^{i} \wedge d x^{j}$ автодуальна или антиавтодуальна.

Доказательств о. Выпишем все $T_{i j}(28)$ при $s=4\left(\eta_{i i}=\eta^{i i}=1\right)$ :

$$
\begin{array}{ll}
T_{11}=-\left(E_{1}\right)^{2}-\left(E_{2}\right)^{2}-\left(E_{3}\right)^{2}+\left(H_{1}\right)^{2}+\left(H_{2}\right)^{2}+\left(H_{3}\right)^{2}, \\
T_{22}=-\left(E_{1}\right)^{2}+\left(E_{2}\right)^{2}+\left(E_{3}\right)^{2}+\left(H_{1}\right)^{2}-\left(H_{2}\right)^{2}-\left(H_{3}\right)^{2}, \\
T_{33}=\left(E_{1}\right)^{2}-\left(E_{2}\right)^{2}+\left(E_{3}\right)^{2}-\left(H_{1}\right)^{2}+\left(H_{2}\right)^{2}-\left(H_{3}\right)^{2}, \\
T_{44}=\left(E_{1}\right)^{2}+\left(E_{2}\right)^{2}-\left(E_{3}\right)^{2}-\left(H_{1}\right)^{2}-\left(H_{2}\right)^{2}+\left(H_{3}\right)^{2}, \\
T_{12}=2\left(E_{2} H_{3}-E_{3} H_{2}\right), & T_{13}=2\left(E_{3} H_{1}-E_{1} H_{3}\right), \\
T_{14}=\left(E_{1} H_{2}-E_{2} H_{1}\right), & T_{23}=2\left(H_{1} H_{2}-E_{1} E_{2}\right), \\
T_{24}=2\left(H_{1} H_{3}-E_{1} E_{3}\right), & T_{34}=2\left(H_{2} H_{3}-E_{2} E_{3}\right) .
\end{array}
$$

Отсюда нетрудно убедиться, что равенство $T_{i j}=0$ при всех $i, j$ возможно только при

$$
E_{1}=\epsilon H_{1}, \quad E_{2}=\epsilon H_{2}, \quad E_{3}=\epsilon H_{3}, \quad \epsilon= \pm 1 .
$$

С помощью (10) и (11) убеждаемся, что $* F= \pm F$. При $s=-4$ вычисления идентичные, т. к. компоненты $T_{i j}$ отличаются лишь знаком. 
При $s=0\left(\eta_{11}=\eta_{22}=-1\right.$, а $\left.\eta_{33}=\eta_{44}=1\right)$ будем иметь

$$
\begin{aligned}
& T_{11}=\left(E_{1}\right)^{2}-\left(E_{2}\right)^{2}-\left(E_{3}\right)^{2}-\left(H_{1}\right)^{2}+\left(H_{2}\right)^{2}+\left(H_{3}\right)^{2}, \\
& T_{22}=\left(E_{1}\right)^{2}+\left(E_{2}\right)^{2}+\left(E_{3}\right)^{2}-\left(H_{1}\right)^{2}-\left(H_{2}\right)^{2}-\left(H_{3}\right)^{2}, \\
& T_{33}=\left(E_{1}\right)^{2}+\left(E_{2}\right)^{2}-\left(E_{3}\right)^{2}-\left(H_{1}\right)^{2}-\left(H_{2}\right)^{2}+\left(H_{3}\right)^{2}, \\
& T_{44}=\left(E_{1}\right)^{2}-\left(E_{2}\right)^{2}+\left(E_{3}\right)^{2}-\left(H_{1}\right)^{2}+\left(H_{2}\right)^{2}-\left(H_{3}\right)^{2}, \\
& T_{12}=2\left(E_{2} H_{3}-E_{3} H_{2}\right), \quad T_{13}=2\left(E_{3} H_{1}+E_{1} H_{3}\right), \\
& T_{14}=-2\left(E_{1} H_{2}+E_{2} H_{1}\right), \quad T_{23}=2\left(H_{1} H_{2}+E_{1} E_{2}\right), \\
& T_{24}=2\left(H_{1} H_{3}+E_{1} E_{3}\right), \quad T_{34}=2\left(E_{2} E_{3}-H_{2} H_{3}\right) .
\end{aligned}
$$

Если все $T_{i j}=0$, то

$$
E_{1}=\epsilon H_{1}, \quad E_{2}=-\epsilon H_{2}, \quad E_{3}=-\epsilon H_{3}, \quad \epsilon= \pm 1 .
$$

С помощью (10) и (12) снова убеждаемся, что $* F= \pm F$, что и доказывает лемму.

Применим этот результат для решений уравнений Янга-Миллса (17)-(19) на 4-многообразии конформной связности при равенстве нулю тензора Вейля $C_{i k j l}=0$. В этом случае квадратичная форма $\psi=\eta_{i j} \omega^{i} \omega^{j}$ угловой метрики конформно евклидова. Путем калибровочного преобразования перенормировки она может быть преобразована в евклидову квадратичную форму. Следовательно, ее тензор Римана $R_{i k j l}$ станет нулевым. Значит, нулевым будет и тензор Баха (24). Поэтому уравнение Янга-Миллса (26) сведется к $T_{i j}=0$, где $T_{i j}$ - тензор энергии-импульса тензора $b_{[i j]}$, компонент 2-формы $(6)_{1}$

$$
\Phi_{0}^{0}=\omega_{i} \wedge \omega^{i}=\frac{1}{2} b_{[i j]} \omega^{j} \wedge \omega^{i}
$$

Отсюда в случае сигнатуры $s= \pm 2$, как мы показали в начале этого раздела, следует, что $b_{[i j]}=0$, т. е. $\Phi_{0}^{0}=0$. Так как $R_{i j}=0$, в силу $(22)$ $b_{(i j)}=0$. Поэтому все $b_{i j}=0$, следовательно, $\omega_{i}=b_{i k} \omega^{k}=0$. Из $(6)_{2,4}$ с учетом $R_{i}^{j}=\frac{1}{2} R_{i k l}^{j} \omega^{k} \wedge \omega^{l}=0$ следует, что $\Phi_{i}=\Phi_{i}^{j}=0$. Поэтому все элементы матрицы кривизны (5) равны нулю, и уравнения (17)-(19) удовлетворяются тривиальным образом. Итак, доказана

Теорема 1. В случае равенства нулю тензора Вейля $C_{i k j l}=0$ и при сигнатурах $s= \pm 2$ уравнения Янга-Миллса на 4-многообразиях конформной связности выполняются только при нулевой матрице кривизны.

Теперь исследуем пространства Янга-Миллса при нулевом тензоре Вейля для остальных сигнатур $s= \pm 4 ; 0$. В этих случаях тензор Баха снова будет нулевым, и поэтому уравнение (23) сведется к $T_{i j}=0$, где $T_{i j}$ - тензор энергии-импульса тензора $b_{[i j]}$, компонент 2-формы $\Phi_{0}^{0}=\frac{1}{2} b_{[i j]} \omega^{j} \wedge \omega^{i}$. Но теперь из $T_{i j}=0$ не вытекает равенство $\Phi_{0}^{0}=0$, а, согласно лемме, следует лишь, что

$$
* \Phi_{0}^{0}= \pm \Phi_{0}^{0} .
$$

Величины $b_{i j}$ уже не нулевые, а лишь кососимметричные, т. е. $b_{(i j)}=0$, $b_{i j}=\frac{1}{2} b_{[i j]}$. Из $(6)_{4}$ с учетом $R_{i}^{j}=0$ получим

$$
\Phi_{i}^{j}=\omega^{j} \wedge \omega_{i}+\eta^{j m} \eta_{i k} \omega_{m} \wedge \omega^{k}=b_{i k} \omega^{j} \wedge \omega^{k}+\eta^{j m} \eta_{i k} b_{m p} \omega^{p} \wedge \omega^{k} .
$$


Таким образом, все элементы матрицы $\Phi(5)$ конформной кривизны выражаются через компоненты $b_{i j}=\frac{1}{2} b_{[i j]} 2$-формы заряда $\Phi_{0}^{0}$ :

$$
\begin{gathered}
\Phi_{0}^{0}=b_{i j} \omega^{j} \wedge \omega^{i}, \quad \Phi_{i}^{j}=b_{i k} \omega^{j} \wedge \omega^{k}+\eta^{j m} \eta_{i k} b_{m p} \omega^{p} \wedge \omega^{k} \\
\Phi_{i}=\nabla \omega_{i}=\nabla\left(b_{i j} \omega^{j}\right)=b_{i j \mid k} \omega^{k} \wedge \omega^{j}
\end{gathered}
$$

Последнее равенство следует из (6) 3 и (14).

Покажем, что из равенства (30) вытекает такое же равенство и для всей матрицы кривизны

$$
* \Phi= \pm \Phi .
$$

Доказательство одинаковое как для сигнатур $s= \pm 4$, так и для сигнатуры $s=0$. Из $(31)_{1}$ и $b_{(i j)}=0$ следует

$$
\begin{aligned}
\Phi_{0}^{0}=-2\left(b_{12} \omega^{1} \wedge \omega^{2}+b_{13} \omega^{1} \wedge \omega^{3}+\right. & b_{14} \omega^{1} \wedge \omega^{4}+ \\
& \left.+b_{23} \omega^{2} \wedge \omega^{3}+b_{24} \omega^{2} \wedge \omega^{4}+b_{34} \omega^{3} \wedge \omega^{4}\right) .
\end{aligned}
$$

Будем для определенности считать, что $s= \pm 4$ и $* \Phi_{0}^{0}=\Phi_{0}^{0}$. Тогда, согласно (11),

$$
\begin{aligned}
* \Phi_{0}^{0}=-2\left(b_{12} \omega^{3} \wedge \omega^{4}-b_{13} \omega^{2} \wedge \omega^{4}\right. & +b_{14} \omega^{2} \wedge \omega^{3}+ \\
& \left.+b_{23} \omega^{1} \wedge \omega^{4}-b_{24} \omega^{1} \wedge \omega^{3}+b_{34} \omega^{1} \wedge \omega^{2}\right) .
\end{aligned}
$$

В компонентах $* \Phi_{0}^{0}=\Phi_{0}^{0}$ означает, что

$$
b_{23}=b_{14}, \quad b_{34}=b_{12}, \quad b_{24}=-b_{13},
$$

тогда

$$
\begin{aligned}
\Phi_{0}^{0}=-2\left(b_{12} \omega^{1} \wedge \omega^{2}+b_{13} \omega^{1} \wedge \omega^{3}+b_{14} \omega^{1} \wedge \omega^{4}+\right. \\
\left.+b_{14} \omega^{2} \wedge \omega^{3}-b_{13} \omega^{2} \wedge \omega^{4}+b_{12} \omega^{3} \wedge \omega^{4}\right) .
\end{aligned}
$$

Так как выполняются уравнения Максвелла (17) $d \Phi_{0}^{0}=0$, это приводит к следующим соотношениям на компоненты $b_{12}, b_{13}$ и $b_{14}$ :

$$
\begin{array}{ll}
b_{12 \mid 3}-b_{13 \mid 2}+b_{14 \mid 1}=0, & b_{12 \mid 4}-b_{14 \mid 2}-b_{13 \mid 1}=0 \\
b_{13 \mid 4}-b_{14 \mid 3}+b_{12 \mid 1}=0, & b_{14 \mid 4}+b_{13 \mid 3}+b_{12 \mid 2}=0 .
\end{array}
$$

Покажем, например, что $* \Phi_{1}=\Phi_{1}$. В силу $(31)_{3}$

$$
\begin{aligned}
\Phi_{1}=b_{12 \mid 1} \omega^{1} \wedge \omega^{2}+b_{13 \mid 1} \omega^{1} & \wedge \omega^{3}+b_{14 \mid 1} \omega^{1} \wedge \omega^{4}+\left(b_{13 \mid 2}-b_{12 \mid 3}\right) \omega^{2} \wedge \omega^{3}+ \\
& +\left(b_{14 \mid 2}-b_{12 \mid 4}\right) \omega^{2} \wedge \omega^{4}+\left(b_{14 \mid 3}-b_{13 \mid 4}\right) \omega^{3} \wedge \omega^{4} .
\end{aligned}
$$

Из (10) и (11) имеем

$$
\begin{array}{r}
* \Phi_{1}=b_{12 \mid 1} \omega^{3} \wedge \omega^{4}-b_{13 \mid 1} \omega^{2} \wedge \omega^{4}+b_{14 \mid 1} \omega^{2} \wedge \omega^{3}+\left(b_{13 \mid 2}-b_{12 \mid 3}\right) \omega^{1} \wedge \omega^{4}- \\
-\left(b_{14 \mid 2}-b_{12 \mid 4}\right) \omega^{1} \wedge \omega^{3}+\left(b_{14 \mid 3}-b_{13 \mid 4}\right) \omega^{1} \wedge \omega^{2} .
\end{array}
$$


В силу равенств $(35)$ получаем $* \Phi_{1}=\Phi_{1}$. Аналогично доказывается $* \Phi_{i}=\Phi_{i}$ для любых $i$.

Докажем теперь, что $* \Phi_{i}^{j}=\Phi_{i}^{j}$. Формула (16) в подробной записи дает

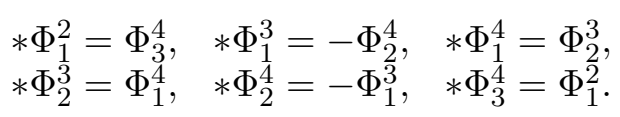

Из $(31)_{2}$ и (33) имеем

$$
\begin{aligned}
& \Phi_{1}^{2}=b_{13} \omega^{2} \wedge \omega^{3}+b_{14} \omega^{2} \wedge \omega^{4}-b_{14} \omega^{1} \wedge \omega^{3}+b_{13} \omega^{1} \wedge \omega^{4}, \\
& \Phi_{3}^{4}=b_{13} \omega^{1} \wedge \omega^{4}+b_{14} \omega^{2} \wedge \omega^{4}-b_{14} \omega^{1} \wedge \omega^{3}+b_{13} \omega^{2} \wedge \omega^{3},
\end{aligned}
$$

т. е. $\Phi_{1}^{2}=\Phi_{3}^{4}$. Отсюда в силу (36) получим $* \Phi_{1}^{2}=\Phi_{1}^{2}$ и $* \Phi_{3}^{4}=\Phi_{3}^{4}$. Аналогично проверяются все остальные равенства $* \Phi_{i}^{j}=\Phi_{i}^{j}$. Итак, доказана формула $(32)$ и

Теорема 2. В случае равенства нулю тензора Вейля $C_{i k j l}=0$ и при сигнатурах $s= \pm 4 ; 0$ уравнения Янга-Миллса на 4-многообразиях конформной связности имеют только автодуальные или антиавтодуальные решения, $* \Phi= \pm \Phi$, причем вся матрииа кривизны $\Phi$ вычисляется только через компоненты $b_{[i j]} 2$-формы заряда $\Phi_{0}^{0}=\frac{1}{2} b_{[i j]} \omega^{j} \wedge \omega^{i}$.

Уравнения Янга-Миллса (13) при условии (32) выполняются автоматически вследствие тождеств Бианки (7), поэтому уравнения Янга-Миллса сводятся к условиям дуальности, имеющим вид (33) и (35), на шесть кососимметричных функций $b_{i j}$ (при сигнатуре $s=0$ или антиавтодуальности матрицы $\Phi$ уравнения (33) и (35) заменяются аналогичными, получить которые не составляет никакого труда; мы их не записываем).

3. Автодуальные и антиавтодуальные решения уравнений Максвелла в евклидовом и псевдоевклидовом (сигнатура 0) 4-мерном пространстве. В предыдущем разделе мы доказали, что система уравнений Янга-Миллса (17)-(19) при сигнатуре угловой метрики $s= \pm 4$ и нулевом тензоре Вейля сводится к уравнениям (35) на три коэффициента 2-формы заряда $\Phi_{0}^{0}$, заданной (34). Мы откалибровали квадратичную форму $\psi=\eta_{i j} \omega^{i} \omega^{j}$ угловой метрики на каждой карте так, чтобы она стала евклидовой на любой карте. Далее, уже локально, в окрестности фиксированной точки можно выбрать такие координаты $u^{1}, u^{2}, u^{3}, u^{4}$, чтобы формы Кристоффеля $\omega_{i}^{j}$ стали нулевыми и $\omega^{i}=d u^{i}$. Тогда в уравнениях (35) ковариантные производные заменятся обычными частными производными по координатам. Полагая $b_{12}=E_{1}, b_{13}=E_{2}, b_{14}=E_{3}$, перепишем эти уравнения в виде

$$
\begin{array}{ll}
\frac{\partial E_{1}}{\partial u^{3}}-\frac{\partial E_{2}}{\partial u^{2}}+\frac{\partial E_{3}}{\partial u^{1}}=0, & \frac{\partial E_{1}}{\partial u^{1}}+\frac{\partial E_{2}}{\partial u^{4}}-\frac{\partial E_{3}}{\partial u^{3}}=0, \\
\frac{\partial E_{1}}{\partial u^{4}}-\frac{\partial E_{2}}{\partial u^{1}}-\frac{\partial E_{3}}{\partial u^{2}}=0, & \frac{\partial E_{1}}{\partial u^{2}}+\frac{\partial E_{2}}{\partial u^{3}}+\frac{\partial E_{3}}{\partial u^{4}}=0 .
\end{array}
$$

Напомним, что (37) является просто компонентной записью уравнения Максвелла (17) $d \Phi_{0}^{0}=0$, где 2 -форма $\Phi_{0}^{0}-$ автодуальная, $* \Phi_{0}^{0}=\Phi_{0}^{0}$, поэтому второе уравнение (17) совпадает с первым. Таким образом, решения уравнений (37) дают нам все автодуальные решения уравнений Максвелла в евклидовом 4-мерном пространстве. Система (37) переопределенная, поэтому 
в первую очередь надо убедиться в ее разрешимости. Удобнее исходить из уравнения $d \Phi_{0}^{0}=0$, которое в подробной записи исходя из $(34)$ имеет вид

$$
\begin{aligned}
& d E_{1} \wedge\left(d u^{1} \wedge d u^{2}+d u^{3} \wedge d u^{4}\right)+ d E_{2} \wedge\left(d u^{1} \wedge d u^{3}-d u^{2} \wedge d u^{4}\right)+ \\
&+d E_{3} \wedge\left(d u^{1} \wedge d u^{4}+d u^{2} \wedge d u^{3}\right)=0 .
\end{aligned}
$$

Имеем одно внешнее уравнение 3-го порядка на три искомые функции. Согласно стандартной схеме исследования внешних систем уравнений (см. [7]), обозначая за $s_{i}$ число внешних уравнений порядка $i$, где $i=1,2,3,4$, для числа Картана $Q$ получаем

$$
Q=s_{1}+2 s_{2}+3 s_{3}+4 s_{4}=3 \text {. }
$$

Число $N$ всех параметров также равно 3. Поэтому $Q=N$, следовательно, уравнение (38) в инволюции, и решения уравнения (38) существуют с произволом в одну функцию трех аргументов.

Система (37) аналогична системе уравнений Коши-Римана для голоморфной функции одной комплексной переменной. Аналогия, во-первых, состоит в том, что как у голоморфной функции вещественная и мнимая части гармоничны, так и функции $E_{1}, E_{2}, E_{3}$, удовлетворяющие (37), также гармоничны. Действительно, дифференцируя первое уравнение по $u^{3}$, второе - по $u^{1}$, третье - по $u^{4}$, четвертое - по $u^{2}$ и складывая, получим

$$
\frac{\partial^{2} E_{1}}{\left(\partial u^{1}\right)^{2}}+\frac{\partial^{2} E_{1}}{\left(\partial u^{2}\right)^{2}}+\frac{\partial^{2} E_{1}}{\left(\partial u^{3}\right)^{2}}+\frac{\partial^{2} E_{1}}{\left(\partial u^{4}\right)^{2}}=0 .
$$

Аналогично доказывается гармоничность функций $E_{2}$ и $E_{3}$. Во-вторых, голоморфную функцию можно восстановить с точностью до константы, зная вещественную и мнимую части. Это же верно и для системы (37). Зная одну из функций $E_{1}, E_{2}, E_{3}$, остальные две определяются через нее с некоторым произволом, который мы оценим. Покажем, как восстановить функции $E_{1}$ и $E_{2}$, зная гармоническую функцию $E_{3}$. В этом случае уравнение (38) не будет в инволюции, так как $Q=3, N=2$, поэтому уравнение (38) надо продолжать. Но короче получится, если исходить из системы (37). Умножим первое уравнение на $d u^{3}$, второе - на $d u^{1}$, третье - на $d u^{4}$, четвертое - на $d u^{2}$ и сложим:

$$
\begin{aligned}
d E_{1}-\frac{\partial E_{2}}{\partial u^{2}} d u^{3}-\frac{\partial E_{2}}{\partial u^{1}} d u^{4} & +\frac{\partial E_{2}}{\partial u^{4}} d u^{1}+\frac{\partial E_{2}}{\partial u^{3}} d u^{2}+ \\
+ & \frac{\partial E_{3}}{\partial u^{1}} d u^{3}-\frac{\partial E_{3}}{\partial u^{2}} d u^{4}-\frac{\partial E_{3}}{\partial u^{3}} d u^{1}+\frac{\partial E_{3}}{\partial u^{4}} d u^{2}=0 .
\end{aligned}
$$

Дифференцируя это уравнение внешне и приравнивая к нулю коэффициенты перед $d u^{i} \wedge d u^{j}$, получим пять дифференциальных уравнений в частных производных второго порядка на функцию $E_{2}$. Но в силу предположенной 
гармоничности функции $E_{3}$ из них независимых только четыре:

$$
\begin{aligned}
\frac{\partial^{2} E_{2}}{\partial u^{1} \partial u^{2}} & =-\frac{\partial^{2} E_{2}}{\partial u^{3} \partial u^{4}}+\frac{\partial^{2} E_{3}}{\left(\partial u^{1}\right)^{2}}+\frac{\partial^{2} E_{3}}{\left(\partial u^{3}\right)^{2}} \\
\frac{\partial^{2} E_{2}}{\left(\partial u^{2}\right)^{2}} & =-\frac{\partial^{2} E_{2}}{\left(\partial u^{3}\right)^{2}}+\frac{\partial^{2} E_{3}}{\partial u^{1} \partial u^{2}}+\frac{\partial^{2} E_{3}}{\partial u^{3} \partial u^{4}} \\
\frac{\partial^{2} E_{2}}{\left(\partial u^{1}\right)^{2}} & =-\frac{\partial^{2} E_{2}}{\left(\partial u^{4}\right)^{2}}-\frac{\partial^{2} E_{3}}{\partial u^{1} \partial u^{2}}+\frac{\partial^{2} E_{3}}{\partial u^{3} \partial u^{4}}, \\
\frac{\partial^{2} E_{2}}{\partial u^{4} \partial u^{2}} & =-\frac{\partial^{2} E_{2}}{\partial u^{3} \partial u^{1}}+\frac{\partial^{2} E_{3}}{\partial u^{1} \partial u^{4}}+\frac{\partial^{2} E_{3}}{\partial u^{3} \partial u^{2}}
\end{aligned}
$$

Легко проверить, что равенства

$$
\begin{array}{lll}
\frac{\partial}{\partial u^{2}}\left(\frac{\partial^{2} E_{2}}{\partial u^{1} \partial u^{2}}\right)=\frac{\partial}{\partial u^{1}}\left(\frac{\partial^{2} E_{2}}{\left(\partial u^{2}\right)^{2}}\right), & \frac{\partial}{\partial u^{1}}\left(\frac{\partial^{2} E_{2}}{\partial u^{1} \partial u^{2}}\right)=\frac{\partial}{\partial u^{2}}\left(\frac{\partial^{2} E_{2}}{\left(\partial u^{1}\right)^{2}}\right), \\
\frac{\partial}{\partial u^{4}}\left(\frac{\partial^{2} E_{2}}{\partial u^{1} \partial u^{2}}\right)=\frac{\partial}{\partial u^{1}}\left(\frac{\partial^{2} E_{2}}{\partial u^{4} \partial u^{2}}\right), & \frac{\partial}{\partial u^{4}}\left(\frac{\partial^{2} E_{2}}{\left(\partial u^{2}\right)^{2}}\right)=\frac{\partial}{\partial u^{2}}\left(\frac{\partial^{2} E_{2}}{\partial u^{4} \partial u^{2}}\right)
\end{array}
$$

совпадают, соответственно, с производной $\frac{\partial}{\partial u^{3}}$ от 4-го уравнения $(40), \frac{\partial}{\partial u^{4}}$ от 4-го уравнения, $\frac{\partial}{\partial u^{3}}$ от 3 -го уравнения и $\frac{\partial}{\partial u^{3}}$ от 1 -го уравнения. Следовательно, система (40) пассивная. Согласно принципу экономии начальных условий (см. [7]), параметрическая часть функции $E_{2}$ получается из ее разложения в степенной ряд по степеням $u^{i}-u_{0}^{i}, i=1,2,3,4$, путем вычеркивания членов, делящихся на $\left(u^{1}-u_{0}^{1}\right)^{2},\left(u^{2}-u_{0}^{2}\right)^{2},\left(u^{1}-u_{0}^{1}\right)\left(u^{2}-u_{0}^{2}\right),\left(u^{2}-u_{0}^{2}\right)\left(u^{4}-u_{0}^{4}\right)$, и имеет вид

$$
f_{1}\left(u^{3}, u^{4}\right)+\left(u^{1}-u_{0}^{1}\right) \cdot f_{2}\left(u^{3}, u^{4}\right)+\left(u^{2}-u_{0}^{2}\right) \cdot f_{3}\left(u^{3}\right),
$$

где $f_{1}, f_{2}, f_{3}$ - произвольные аналитические функции своих аргументов. Итак, система (40) имеет единственное решение $E_{2}\left(u^{1}, u^{2}, u^{3}, u^{4}\right)$ при следующих начальных условиях:

$$
\begin{aligned}
& E_{2}\left(u_{0}^{1}, u_{0}^{2}, u^{3}, u^{4}\right)=f_{1}\left(u^{3}, u^{4}\right), \\
& \frac{\partial E_{2}}{\partial u^{1}}\left(u_{0}^{1}, u_{0}^{2}, u^{3}, u^{4}\right)=f_{2}\left(u^{3}, u^{4}\right), \\
& \frac{\partial E_{2}}{\partial u^{2}}\left(u_{0}^{1}, u_{0}^{2}, u^{3}, u^{4}\right)=f_{3}\left(u^{3}\right) .
\end{aligned}
$$

Найдя функцию $E_{2}$, из уравнения (39) находим функцию $E_{1}$ с точностью до константы.

Тот факт, что одну из функций $E_{1}, E_{2}, E_{3}$ можно взять произвольной гармонической, позволяет получить большое число конкретных решений системы (37). Например, положим $E_{3}=$ const. Тогда система (37) примет вид

$$
\frac{\partial E_{1}}{\partial u^{3}}-\frac{\partial E_{2}}{\partial u^{2}}=0, \quad \frac{\partial E_{1}}{\partial u^{1}}+\frac{\partial E_{2}}{\partial u^{4}}=0, \quad \frac{\partial E_{1}}{\partial u^{4}}-\frac{\partial E_{2}}{\partial u^{1}}=0, \quad \frac{\partial E_{1}}{\partial u^{2}}+\frac{\partial E_{2}}{\partial u^{3}}=0 .
$$

Функции $E_{1}$ и $E_{2}$ удовлетворяют условиям Коши-Римана как по переменным $u^{4}, u^{1}$, так и по переменным $u^{3}, u^{2}$. Отсюда следует, что для любых 
голоморфных функций $f\left(u^{3}+i \cdot u^{2}\right)$ и $g\left(u^{4}+i \cdot u^{1}\right)$, где $i-$ мнимая единица, система (41) будет иметь решение

$$
E_{1}\left(u^{1}, u^{2}, u^{3}, u^{4}\right)=\operatorname{Re} f+\operatorname{Re} g, \quad E_{2}\left(u^{1}, u^{2}, u^{3}, u^{4}\right)=\operatorname{Im} f+\operatorname{Im} g .
$$

Понятно, что формулы (42) не дают общего решения системы (41), это просто пример.

Если 2-форма $\Phi_{0}^{0}-$ антиавтодуальная и $s= \pm 4$, то уравнение Максвелла $d \Phi_{0}^{0}=0$ сводится к следующей системе дифференциальных уравнений

$$
\begin{array}{ll}
\frac{\partial E_{1}}{\partial u^{3}}-\frac{\partial E_{2}}{\partial u^{2}}-\frac{\partial E_{3}}{\partial u^{1}}=0, & \frac{\partial E_{1}}{\partial u^{4}}+\frac{\partial E_{2}}{\partial u^{1}}-\frac{\partial E_{3}}{\partial u^{2}}=0 \\
\frac{\partial E_{1}}{\partial u^{1}}-\frac{\partial E_{2}}{\partial u^{4}}+\frac{\partial E_{3}}{\partial u^{3}}=0, & \frac{\partial E_{1}}{\partial u^{2}}+\frac{\partial E_{2}}{\partial u^{3}}+\frac{\partial E_{3}}{\partial u^{4}}=0 .
\end{array}
$$

У этой системы все три функции $E_{1}, E_{2}, E_{3}$ гармонические. Другие свойства такие же, как у системы (37) для автодуальной формы $\Phi_{0}^{0}$.

Обратимся теперь к сигнатуре $s=0$. Если $* \Phi_{0}^{0}=\Phi_{0}^{0}$, то

$$
\begin{aligned}
\Phi_{0}^{0}=-2\left(E_{1} \omega^{1} \wedge \omega^{2}+E_{2} \omega^{1} \wedge \omega^{3}+\right. & E_{3} \omega^{1} \wedge \omega^{4}- \\
& \left.-E_{3} \omega^{2} \wedge \omega^{3}+E_{2} \omega^{2} \wedge \omega^{4}+E_{1} \omega^{3} \wedge \omega^{4}\right) .
\end{aligned}
$$

Уравнение $d \Phi_{0}^{0}=0$ проводит к системе

$$
\begin{array}{ll}
\frac{\partial E_{1}}{\partial u^{3}}-\frac{\partial E_{2}}{\partial u^{2}}-\frac{\partial E_{3}}{\partial u^{1}}=0, & \frac{\partial E_{1}}{\partial u^{4}}-\frac{\partial E_{2}}{\partial u^{1}}-\frac{\partial E_{3}}{\partial u^{2}}=0 \\
\frac{\partial E_{1}}{\partial u^{1}}+\frac{\partial E_{2}}{\partial u^{4}}-\frac{\partial E_{3}}{\partial u^{3}}=0, & \frac{\partial E_{1}}{\partial u^{2}}+\frac{\partial E_{2}}{\partial u^{3}}-\frac{\partial E_{3}}{\partial u^{4}}=0 .
\end{array}
$$

Дифференцируя первое уравнение по $u^{3}$, второе - по $u^{4}$, третье - по $u^{1}$, четвертое - по $u^{2}$ и складывая первое с четвертым и вычитая второе и третье, получим

$$
\frac{\partial^{2} E_{1}}{\left(\partial u^{3}\right)^{2}}+\frac{\partial^{2} E_{1}}{\left(\partial u^{2}\right)^{2}}-\frac{\partial^{2} E_{1}}{\left(\partial u^{1}\right)^{2}}-\frac{\partial^{2} E_{1}}{\left(\partial u^{4}\right)^{2}}=0
$$

Этому же уравнению удовлетворяют и функции $E_{2}$ и $E_{3}$. Обратим внимание, что квадратичная форма псевдометрики имеет другие знаки:

$$
\psi=-\left(d u^{1}\right)^{2}-\left(d u^{2}\right)^{2}+\left(d u^{3}\right)^{2}+\left(d u^{4}\right)^{2} .
$$

Как и система (37), система уравнений (44) совместна и допускает решение с произволом в одну функцию трех аргументов.

Отметим, что каждая из систем (37) и (43) симметрична относительно перестановок функций $E_{1}, E_{2}, E_{3}$, а система (44)- нет. Например, если положить $E_{3}=$ const, то система (44) совпадет с (41), а поэтому будет иметь место и решение (42). Но если положить $E_{2}=$ const, то получится совсем другая система дифференциальных уравнений в частных производных:

$$
\frac{\partial E_{1}}{\partial u^{3}}-\frac{\partial E_{3}}{\partial u^{1}}=0, \quad \frac{\partial E_{1}}{\partial u^{4}}-\frac{\partial E_{3}}{\partial u^{2}}=0, \quad \frac{\partial E_{1}}{\partial u^{1}}-\frac{\partial E_{3}}{\partial u^{3}}=0, \quad \frac{\partial E_{1}}{\partial u^{2}}-\frac{\partial E_{3}}{\partial u^{4}}=0 .
$$


Примером ее решения может служить следующая пара функций:

$$
\begin{aligned}
E_{1}\left(u^{1}, u^{2}, u^{3}, u^{4}\right)= & f\left(u^{1}+u^{3}\right)+ \\
& +g\left(u^{1}-u^{3}\right)+h\left(u^{2}+u^{4}\right)+p\left(u^{2}-u^{4}\right), \\
E_{3}\left(u^{1}, u^{2}, u^{3}, u^{4}\right)= & f\left(u^{1}+u^{3}\right)- \\
& -g\left(u^{1}-u^{3}\right)+h\left(u^{2}+u^{4}\right)-p\left(u^{2}-u^{4}\right),
\end{aligned}
$$

где $f, g, h, p$ - произвольные дифференцируемые функции от одной переменной. Такого решения не может иметь система (41).

Наконец, при условии антиавтодуальности $* \Phi_{0}^{0}=-\Phi_{0}^{0}$ система (44) заменится на

$$
\begin{array}{ll}
\frac{\partial E_{1}}{\partial u^{3}}-\frac{\partial E_{2}}{\partial u^{2}}+\frac{\partial E_{3}}{\partial u^{1}}=0, & \frac{\partial E_{1}}{\partial u^{4}}-\frac{\partial E_{2}}{\partial u^{1}}-\frac{\partial E_{3}}{\partial u^{2}}=0, \\
\frac{\partial E_{1}}{\partial u^{1}}-\frac{\partial E_{2}}{\partial u^{4}}+\frac{\partial E_{3}}{\partial u^{3}}=0, & \frac{\partial E_{1}}{\partial u^{2}}-\frac{\partial E_{2}}{\partial u^{3}}-\frac{\partial E_{3}}{\partial u^{4}}=0 .
\end{array}
$$

На этот раз каждая из функций $E_{i}$ удовлетворяет уравнению

$$
-\frac{\partial^{2} E_{i}}{\left(\partial u^{1}\right)^{2}}-\frac{\partial^{2} E_{i}}{\left(\partial u^{2}\right)^{2}}+\frac{\partial^{2} E_{i}}{\left(\partial u^{3}\right)^{2}}+\frac{\partial^{2} E_{i}}{\left(\partial u^{4}\right)^{2}}=0 .
$$

У этого уравнения знаки слагаемых такие же, как у псевдометрики (45). При $E_{3}=$ const система (47) имеет решения, аналогичные (46), а при $E_{2}=$ const аналогичные (41) (с точностью до нумерации координат и искомых функций).

4. Уравнения Янга-Миллса для случая угловой метрики, конформной метрике Эйнштейна. Пусть теперь квадратичная форма $\eta_{i j} \omega^{i} \omega^{j}$ угловой метрики конформна метрике Эйнштейна. Калибровочным преобразованием перенормировки мы добьемся, чтобы угловая метрика удовлетворяла уравнению Эйнштейна

$$
R_{i j}=\varkappa \eta_{i j} .
$$

Из уравнения (22) с учетом $R=6 b$ найдем

$$
b_{(i j)}=\frac{1}{3} \varkappa \eta_{i j}
$$

Из формулы (25) получаем

$$
P_{i j}=-\frac{1}{6} \varkappa \eta_{i j}
$$

Учитывая свойство тензора Вейля $\eta^{m n} C_{i m j n}=0$, из формул $(50)$ и $(24)$ получаем для тензора Баха $B_{i j}=0$. Отсюда в силу (26) для тензора энергииимпульса заряда $\Phi_{0}^{0}$ получаем $T_{i j}=0$. Как мы уже показали, при сигнатуре $s= \pm 2$ и сам заряд равен нулю:

$$
\Phi_{0}^{0}=0
$$

а при сигнатурах $s= \pm 4 ; 0$ будет

$$
* \Phi_{0}^{0}= \pm \Phi_{0}^{0} .
$$


В случае $s= \pm 2$ из (51) и (29) следует $b_{[i j]}=0$ и (49) превращается в

$$
b_{i j}=\frac{1}{6} \varkappa \eta_{i j}
$$

Как известно, из (48) следует, что $\varkappa=$ const, поэтому из $(31)_{3},(53)$ и (14) имеем

$$
\Phi_{i}=\nabla\left(b_{i j} \omega^{j}\right)=\frac{1}{6} \varkappa \eta_{i j} \nabla \omega^{j}=\frac{1}{6} \varkappa \eta_{i j} \Phi^{j}=0 .
$$

Структурная формула для основного тензора

$$
\Phi_{i j m n}=C_{i j m n}+\frac{1}{2} \eta_{i j} \circ b_{[m n]},
$$

доказанная в [8, стр. 400], в силу $b_{[i j]}=0$ сводится к равенству

$$
\Phi_{i j m n}=C_{i j m n}
$$

Итак, при $s= \pm 2$ и условии (48) уравнения Янга-Миллса (17)-(19) равносильны (51) и (53). Следовательно, в матрице кривизны Ф не равны нулю лишь внешние 2-формы $\Phi_{i}^{j}$, которые выражаятся толъко через тензор Вейля. Иначе говоря, это нормальное пространство Картана с нулевым тензором Баха. Равенство нулю тензора Баха есть критерий выполнимости в нормальном пространстве Картана уравнений Янга-Миллса при любой сигнатуре метрики. Но из равенства нулю тензора Баха не следует конформности угловой метрики $\psi$ метрике Эйнштейна (см. [9]).

Совсем другая ситуация возникает при сигнатурах $s= \pm 4 ; 0$. В этих случаях вместо (51) выполняется (52). Заряд $\Phi_{0}^{0}$ уже может быть ненулевым. Основной тензор выражается через тензор Вейля и заряд по формуле (54). Имеем также с помощью (49)

$$
\omega_{i} \stackrel{\text { def }}{=} b_{i j} \omega^{j}=\frac{1}{2} b_{(i j)} \omega^{j}+\frac{1}{2} b_{[i j]} \omega^{j}=\frac{1}{6} \varkappa \eta_{i j} \omega^{j}+\frac{1}{2} b_{[i j]} \omega^{j} .
$$

Первое слагаемое при внешнем ковариантном дифференцировании дает нуль, поэтому для внешних 2-форм $\Phi_{i}$ из (31) получаем

$$
\Phi_{i}=\nabla \omega_{i}=\frac{1}{2} \nabla\left(b_{[i j]} \omega^{j}\right)=\frac{1}{2} b_{[i j] \mid k} \omega^{k} \wedge \omega^{j} .
$$

Теми же вычислениями, что и в разделе 2 , убеждаемся, что из (52) следует $* \Phi_{i}= \pm \Phi_{i}$. Однако для 2-форм $\Phi_{i}^{j}$ равенства $* \Phi_{i}^{j}= \pm \Phi_{i}^{j}$ не выполняются. В самом деле, если мы в соответствии с формулой (54) введем 2-формы

$$
\sigma_{i j}=\frac{1}{2} C_{i j m n} \omega^{m} \wedge \omega^{n}, \quad \beta_{i j}=\frac{1}{4} \eta_{i j} \circ b_{[m n]} \omega^{m} \wedge \omega^{n}
$$

то легко показать, что $* \beta_{i j}= \pm \beta_{i j}$ (знак \pm один и тот же для всех индексов $i, j)$. Но для выполнения равенства $* \sigma_{i j}= \pm \sigma_{i j}$ нет никаких оснований, так как уравнения Янга-Миллса не меняют алгебраическую структуру тензора Вейля. При выполнении уравнений Янга-Миллса тензор Вейля имеет 
то же число существенных компонент, 10, что и без уравнений Янга-Миллса. Но автодуальные (антиавтодуальные) решения уравнений Янга-Миллса приводят к существенному ограничению на тензор Вейля, т. к. останется только 5 существенных компонент.

Итак, для сигнатур $s= \pm 4 ; 0$ и угловой метрики, конформной метрике Эйнштейна, уравнения Янга-Миллса выполняются, только если:

1) $* \Phi_{0}^{0}= \pm \Phi_{0}^{0}$

2) $\Phi_{i}$ выражаются через $\Phi_{0}^{0}$ и тоже удовлетворяют условиям $* \Phi_{i}= \pm \Phi_{i}$;

3) $\Phi_{i}^{j}$ выражаются через тензор Вейля и заряд по формуле (54).

Выводы. Уравнения Янга-Миллса (13) на 4-многообразиях конформной связности без кручения независимо от сигнатуры угловой метрики $\eta_{i j} \omega^{i} \omega^{j}$ распадаются на три группы уравнений. Первые две группы уравнений - это уравнения Эйнштейна (22) и уравнения Максвелла (17). Третья группа уравнений (26) представляет собой равенство тензора Баха (24) квадратичной формы $\eta_{i j} \omega^{i} \omega^{j}$ и тензора энергии-импульса (28) кососимметричного тензора заряда $b_{[i j]}$. Если пространство конформной связности имеет кручение, то не возникает никаких отдельных групп уравнений, и уравнения Янга-Миллса представляют собой очень сложную систему из 60 дифференциальных уравнений второго порядка в частных производных на 60 неизвестных функций от 4 локальных координат. Авторам пока неизвестно ни одно частное решение уравнений Янга-Миллса в пространстве конформной связности с кручением.

Напротив, в пространстве без кручения при наличии определенных упрощающих условий можно привести некоторые решения уравнений Янга-Миллса в явном виде. Одним из таких упрощающих условий является требование (анти)автодуальности $* \Phi= \pm \Phi$. Если при сигнатуре Минковского $s= \pm 2$ у уравнений Янга-Миллса имеются только тривиальные (анти)автодуальные решения, соответствующие нулевой матрице кривизны $\Phi=0$, то при других сигнатурах существуют и нетривиальные решения, когда $\Phi \neq 0$.

В данной статье доказывается (анти)автодуальность матрицы кривизны $\Phi$ в случае равенства нулю тензора Вейля (теорема 2), а также устанавливается связь между (анти)автодуальностью некоторых компонент матрицы кривизны $\Phi$ и эйнштейновостью метрики $\eta_{i j} \omega^{i} \omega^{j}$.

Конкурирующие интересы. Заявляем, что в отношении авторства и публикации этой статьи конфликта интересов не имеем.

Авторский вклад и ответственность. Все авторы принимали участие в разработке концепции статьи и в написании рукописи. Авторы несут полную ответственность за предоставление окончательной рукописи в печать. Окончательная версия рукописи была одобрена всеми авторами.

Финансирование. Исследование выполнялось без финансирования. 


\section{Библиографический список}

1. Картан Э. Пространства аффинной, проективной и конформной связности. Казань: Казан. ун-т, 1962. 210 с.

2. Кривоносов Л. Н., Лукьянов В. А. Связь уравнений Янга-Миллса с уравнениями Эйнштейна и Максвелла // Журн. СФУ. Сер. Матем. и физ., 2009. Т. 2, № 4. С. 432-448.

3. Atiyah M. F., Hitchin N. J., Singer I. M. Self-duality in four-dimensional Riemannian geometry// Proc. Roy. Soc. London. Series A, 1978. vol.362, no.1711. pp. 425-461. doi: 10.1098/rspa.1978.0143.

4. Singerland I. M., Thorpe J. A. The curvature of 4-dimensional Einstein spaces / Global Analysis: Papers in Honor of K. Kodaira (PMS-29). Princeton: Princeton University Press, 2015. pp. 355-365. doi: 10.1515/9781400871230-021.

5. Sucheta Koshti, Naresh Dadhich The General Self-dual solution of the Einstein Equations, 1994, arXiv: gr-qc/9409046.

6. Ландау Л. Д., Лифшиц Е. М. Теория поля. М.: Наука, 1973. 504 с.

7. Фиников С. П. Метод внешних форм Картана в дифференциальной геометрии. М.: ГИТТЛ, 1948. 432 с.

8. Кривоносов Л. Н., Лукьянов В. А. Уравнения Эйнштейна на четырехмерном многообразии конформной связности без кручения // Журн. СФУ. Сер. Матем. и физ., 2012. T. 5, № 3. С. 393-408.

9. Korzyjński M., Levandowski J. The Normal Conformal Cartan Connection and the Bach Tensor // Class. Quant. Grav., 2003. vol. 20, no. 16. pp. 3745-3764, arXiv: gr-qc/0301096v3. doi: $10.1088 / 0264-9381 / 20 / 16 / 314$. 


\title{
Yang-Mills equations on conformally connected torsion-free 4-manifolds with different signatures
}

\section{N. Krivonosov, V. A. Luk'yanov}

Nizhny Novgorod State Technical University,

24, Minina st., Nizhnii Novgorod, 603600, Russian Federation.

\begin{abstract}
In this paper we study spaces of conformal torsion-free connection of dimension 4 whose connection matrix satisfies the Yang-Mills equations. Here we generalize and strengthen the results obtained by us in previous articles, where the angular metric of these spaces had Minkowski signature. The generalization is that here we investigate the spaces of all possible metric signatures, and the enhancement is due to the fact that additional attention is paid to calculating the curvature matrix and establishing the properties of its components. It is shown that the Yang-Mills equations on 4-manifolds of conformal torsion-free connection for an arbitrary signature of the angular metric are reduced to Einstein's equations, Maxwell's equations and the equality of the Bach tensor of the angular metric and the energy-momentum tensor of the skew-symmetric charge tensor. It is proved that if the Weyl tensor is zero, the Yang-Mills equations have only self-dual or anti-self-dual solutions, i.e the curvature matrix of a conformal connection consists of self-dual or anti-self-dual external 2-forms. With the Minkowski signature (anti)self-dual external 2-forms can only be zero. The components of the curvature matrix are calculated in the case when the angular metric of an arbitrary signature is Einstein, and the connection satisfies the Yang-Mills equations. In the Euclidean and pseudo-Euclidean 4-spaces we give some particular self-dual and anti-self-dual solutions of the Maxwell equations, to which all the Yang-Mills equations are reduced in this case.
\end{abstract}

Keywords: manifolds with conformal connection, curvature, torsion, YangMills equations, Einstein's equations, Maxwell's equations, Hodge operator, (anti)self-dual 2-forms, Weyl tensor, Bach tensor.

Received: $12^{\text {th }}$ October, $2017 /$ Revised: $27^{\text {th }}$ November, $2017 /$

Accepted: $18^{\text {th }}$ December, $2017 /$ First online: $28^{\text {th }}$ December, 2017

\begin{abstract}
Research Article
○ () The content is published under the terms of the Creative Commons Attribution 4.0 International License (http://creativecommons.org/licenses/by/4.0/)

Please cite this article in press as:

Krivonosov L. N., Luk'yanov V. A. Yang-Mills equations on conformally connected torsion-free 4-manifolds with different signatures, Vestn. Samar. Gos. Tekhn. Univ., Ser. Fiz.-Mat. Nauki [J. Samara State Tech. Univ., Ser. Phys. Math. Sci.], 2017, vol. 21, no. 4, pp. 633-650. doi: 10.14498/vsgtu1562 (In Russian).
\end{abstract}

Authors' Details:

Vyacheslav A. Luk'yanov (10) http://orcid.org/0000-0002-7294-0232

Cand. Phys. \& Math. Sci.; Associate Professor; Dept. of Applied Mathematics; e-mail: oxyzt@ya.ru

Leonid N. Krivonosov (D) http://orcid.org/0000-0002-3533-9595

Cand. Phys. \& Math. Sci.; Associate Professor; Dept. of Applied Mathematics;

e-mail: 1.n.krivonosov@gmail.com 
Competing interests. We declare that we have no conflicts of interests with the authorship and publication of this article.

Authors' contributions and responsibilities. Each author has participated in the article concept development and in the manuscript writing. The authors are absolutely responsible for submitting the final manuscript in print. Each author has approved the final version of manuscript.

Funding. The research has not had any funding.

\section{References}

1. Kartan E. Prostranstva affinnoi, proektivnoi i konformnoi sviaznosti [Spaces of affine, projective, and conformal connection]. Kazan', Kazan. un-t, 1962, 210 pp. (In Russian)

2. Krivonosov L. N., Luk'yanov V. A. Connection of Young-Mills Equations with Einstein and Maxwell's Equations, J. Sib. Fed. Univ. Math. Phys., 2009, vol. 2, no. 4, pp. 432-448 (In Russian).

3. Atiyah M. F., Hitchin N. J., Singer I. M. Self-duality in four-dimensional Riemannian geometry, Proc. Roy. Soc. London. Series A, 1978, vol.362, no. 1711, pp. 425-461. doi : 10.1098/rspa.1978.0143.

4. Singerland I. M., Thorpe J. A. The curvature of 4-dimensional Einstein spaces, In: Global Analysis: Papers in Honor of K. Kodaira (PMS-29). Princeton, Princeton University Press, 2015, pp. 355-365. doi : 10.1515/9781400871230-021.

5. Sucheta Koshti, Naresh Dadhich The General Self-dual solution of the Einstein Equations, 1994, arXiv: gr-qc/9409046.

6. Landau L. D., Lifshits E. M. Teoriia polia [Field theory]. Moscow, Nauka, 1973, 504 pp. (In Russian)

7. Finikov S. P. Metod vneshnikh form Kartana v differentsial'noi geometrii [Method of Cartan's external forms in differential geometry]. Moscow, GITTL, 1948, 432 pp. (In Russian)

8. Krivonosov L. N., Luk'yanov V. A. Einstein's equations on a 4-manifold of conformal torsionfree connection, J. Sib. Fed. Univ. Math. Phys., 2012, vol. 5, no. 3, pp. 393-408 (In Russian).

9. Korzyjński M., Levandowski J. The Normal Conformal Cartan Connection and the Bach Tensor, Class. Quant. Grav., 2003, vol. 20, no. 16, pp. 3745-3764, arXiv: gr-qc/0301096v3. doi : $10.1088 / 0264-9381 / 20 / 16 / 314$. 